\title{
Epiphytic and epixylic bryophyte communities of Holosiivskyi National Nature Park
}

\author{
Viktor A. ONYSHCHENKO ${ }^{1,2}$, Vitaliy M. VIRCHENKO ${ }^{1,2}$ \\ ${ }^{1}$ M.G. Kholodny Institute of Botany, National Academy of Sciences of Ukraine \\ 2 Tereschenkivska Str., Kyiv 01004, Ukraine \\ ${ }^{2}$ Holosiivskyi National Nature Park \\ 6a Henerala Rodymtseva Str., Kyiv 03035, Ukraine \\ labzap@ukr.net \\ vir_chen_ko@ukr.net
}

Onyshchenko V.A., Virchenko V.M. 2020. Epiphytic and epixylic bryophyte communities of Holosiivskyi National Nature Park. Ukrainian Botanical Journal, 77(1): 23-33.

\begin{abstract}
The article presents 80 relevés of bryophyte communities on living trees and dead wood sampled within Holosiivskyi National Nature Park (Kyiv, Ukraine). They are referred to 16 associations of 5 classes (Frullanio dilatatae-Leucodontetea sciuroidis, Cladonio digitatae-Lepidozietea reptantis, Neckeretea complanatae, Hylocomietea splendentis, PlatyhypnidioFontinalietea antipyreticae). Most frequent are communities dominated by Hypnum cupressiforme and H. pallescens of the class Frullanio dilatatae-Leucodontetea sciuroidis (on bark of living trees) and Brachythecium rutabulum of the class Cladonio digitataeLepidozietea reptantis (on dead wood). In mesic forests, trees are usually not inhabited by bryophyte communities. Much more often, they occur in wet forests, near water, and in mesic forests in ravines. Four associations are reported in Ukraine for the first time: Callicladietum haldaniani LeBlanc 1963, Brachythecio rutabuli-Plagiomnietum cuspidati (Felföldy 1941) Plamada 1982, Brachythecio rivularis-Hygrohypnetum luridi Philippi 1965, and Leptodictyo riparii-Hygroamblystegietum varii Hugonnot et Celle 2013.
\end{abstract}

Keywords: bryophyte communities, classification, syntaxonomy, Ukraine

Онищенко В.А. ${ }^{1,2}$, Вірченко В.М. ${ }^{1,2}$ 2020. Епіфітні та епіксильні бріоугруповання Національного природного парку "Голосіївський". Український ботанічний журнал, 77(1): 23-33.

${ }^{1}$ Інститут ботаніки ім. М.Г. Холодного НАН України

вул. Терещенківська 2, Київ 01004, Україна

${ }^{2}$ Національний природний парк "Голосіївський"

вул. Генерала Родимцева 6а, Київ 03035, Україна

Реферат. У статті наведено 80 описів угруповань мохоподібних на корі живих дерев і мертвій деревині, виконаних у Києві (Україна). Всі угруповання зараховано до 16 асоціацій п'яти класів (Frullanio dilatatae-Leucodontetea sciuroidis, Cladonio digitatae-Lepidozietea reptantis, Neckeretea complanatae, Hylocomietea splendentis, Platyhypnidio-Fontinalietea antipyreticae). Найчастіше трапляються угруповання $з$ домінуванням Hypnum cupressiforme і H. pallescens класу Frullanio dilatataeLeucodontetea sciuroidis (на корі живих дерев) і домінуванням Brachythecium rutabulum класу Cladonio digitatae-Lepidozietea reptantis (на мертвій деревині). У мезофільних лісах, зазвичай на деревах немає угруповань бріофітів. Набагато частіше вони трапляються в сирих лісах, поблизу води та в ярах. Чотири асоціації наводяться для України вперше: Callicladietum haldaniani LeBlanc 1963, Brachythecio rutabuli-Plagiomnietum cuspidati (Felföldy 1941) Plamada 1982, Brachythecio rivularisHygrohypnetum luridi Philippi 1965, Leptodictyo riparii-Hygroamblystegietum varii Hugonnot \& Celle 2013.

Ключові слова: класифікація, синтаксономія, угруповання мохоподібних, Україна

(C) 2020 V.A. Onyshchenko, V.M. Virchenko. Published by the M.G. Kholodny Institute of Botany, NAS of Ukraine. This is an open access article under the terms of the Creative Commons Attribution License (http://creativecommons.org/licenses/by/4.0/), which permits use, distribution, and reproduction in any medium, provided the original work is properly cited 
Information about bryophyte vegetation in Ukraine, or more precisely in its Forest-Steppe zone, is presented in publications by S. Gapon (2009, 2012, 2014, 2017), Yu. Gapon (2015, 2017), S. Gapon and Yu. Gapon (2018). Brief descriptions of bryophyte communities in one of the parks of Kyiv city is provided by Virchenko and S. Gapon (2009). In our article, we report new material on this issue and its interpretation. The data were sampled in Holosiivskyi National Nature Park (Kyiv, Ukraine). Vegetation of the park is represented mainly by continental forests of Pinus sylvestris L. (Pyrolo-Pinetea Korneck 1974), deciduous forests dominated by Quercus robur L., Carpinus betulus L., Acer campestre L., Fraxinus excelsior L., Alnus glutinosa (L.) Gaertn. (Carpino-Fagetea sylvaticae Jakucs ex Passarge 1968, Alno-Fraxinetalia excelsioris Passarge 1968, Quercetea pubescentis Doing-Kraft ex Scamoni et Passarge 1959), and Alnus glutinosa swamps (Alnetalia glutinosae Tx. 1937). Bryophyte communities were studied on living and dead trees in 2017-2018. Totally 80 relevés have been sampled in different parts of the national park: Horikhuvatka and Holosiiv valleys in Holosiivskyi Wood, floodplain of the Vita River (Lisnyky Reserve), Teremky Wood, Dachne forestry, Sviatoshynske forestry. Each plot area was $4 \mathrm{dm}^{2}$. A total number of bryophyte species in the relevés is 41. Data were included in the second version (2018) of the database "Vegetation Database of Ukraine and Adjacent Parts of Russia" (GIVD index EUUA-006).

Most relevés were assigned to associations using syntaxonomic arrangement of bryophyte communities by Marstaller (2006). Higher syntaxa are given according to Vegetation of Europe... by Mucina et al. (2016). Names of bryophyte species follow Boiko (2008).

The relevés were referred to 16 associations of 5 classes (constancies of species in higher syntaxa are shown in Table 1):

\section{Frullanio dilatatae-Leucodontetea sciuroidis Mohan 1978}

Orthotrichetalia Hadač in Klika \& Hadač 1944

Leskeion polycarpae Barkman 1958

Leskeetum polycarpae Horvat 1932

Ulotion crispae Ochsner 1928

Pylaisietum polyanthae Gams ex Felföldy 1941

Syntrichion laevipilae Ochsner 1928

Orthotrichetum fallacis von Krusenstjerna 1945

Dicranetalia scoparii Barkman 1958

Dicrano scoparii-Hypnion filiformis Barkman 1958

Platygyrietum repentis LeBlank ex Marstaller 1986
Callicladietum haldaniani LeBlanc 1963

Ptilidio pulcherrimi-Hypnetum pallescentis Herzog 1943 (Ptilidio pulcherrimi-Hypnetum reptile Gapon 2010)

Dicrano montani-Hypnetum cupressiformis

Wiśniewski 1930

Dicrano scoparii-Hypnetum filiformis Barkman 1949

comm. Dicranum tauricum - [FrullanioLeucodontetea]

Cladonio digitatae-Lepidozietea reptantis Ježek \& Vondráček 1962

Brachythecietalia rutabulo-salebrosi Marstaller 1987

Bryo capillaris-Brachythecion rutabuli Lecointe 1975

Brachythecio salebrosi-Amblystegietum juratzkani (Sjögren ex Marstaller 1987) Marstaller 1987

Brachythecio rutabuli-Hypnetum cupressiformis Nörr 1969

Neckeretea complanatae Marstaller 1986

Neckeretalia complanatae Ježek \& Vondráček 1962

Neckerion complanatae Šmarda \& Hadač ex Klika 1946

Anomodontetum attenuati (Barkman 1958) Peciar 1965

Anomodontetum longifolii Waldheim 1944

Plagiomnio cuspidati-Homalietum trichomanoidis (Peciar 1965) Marstaller 1993

Hylocomietea splendentis Gillet ex Marstaller 1992

Hylocomietalia splendentis Gillet ex Vadam 1990

Eurhynchion striati Waldheim 1944

Brachythecio rutabuli-Plagiomnietum cuspidati (Felföldy 1941) Plamada 1982 (Mnietum cuspidati Felföldy 1941)

Climacion dendroidis Ştefureac 1941

Platyhypnidio-Fontinalietea antipyreticae Philippi 1956 Leptodictyetalia riparii Philippi 1956

Brachythecion rivularis Hertel 1974

Brachythecio rivularis-Hygrohypnetum luridi Philippi 1965 (Brachythecietum rivularis Herzog 1943)

Leptodictyo riparii-Hygroamblystegietum varii Hugonnot \& Celle 2013

The class Frullanio dilatatae-Leucodontetea sciuroidis includes predominantly bryophyte communities on the bark of living trees. Order Orthotrichetalia (relevés are presented in Table 2) comprises vegetation of light-demanding mesophilous species on trees with 
Table 1. Synoptic table of epiphytic and epixylic bryophyte communities of Holosiivskyi National Nature Park at the level of order

\begin{tabular}{|c|c|c|c|c|c|c|}
\hline Class & \multicolumn{2}{|c|}{ FRU } & \multirow{2}{*}{$\frac{\text { CLE }}{\mathrm{Bra}}$} & \multirow{2}{*}{$\frac{\mathrm{NEC}}{\mathrm{Nec}}$} & \multirow{2}{*}{$\frac{\text { HYL }}{\text { Hyl }}$} & \multirow{2}{*}{$\frac{\text { PLA }}{\text { Lep }}$} \\
\hline Order & Ort & Dic & & & & \\
\hline Number of plots & 13 & 32 & 11 & 14 & 4 & 6 \\
\hline Orthotrichum pumilum & 46 & . & . & . & . & . \\
\hline Orthotrichum speciosum & 23 & 3 & $\cdot$ & $\cdot$ & $\cdot$ & $\cdot$ \\
\hline Hypnum pallescens & . & 53 & . & . & . & . \\
\hline Orthodicranum montanum & $\cdot$ & 37 & $\cdot$ & $\cdot$ & $\cdot$ & $\cdot$ \\
\hline Platygyrium repens & . & 38 & . & . & . & . \\
\hline Amblystegium serpens & . & $\cdot$ & 27 & . & . & . \\
\hline Brachythecium rutabulum & $\cdot$ & $\cdot$ & 91 & $\cdot$ & 25 & $\cdot$ \\
\hline Homalia trichomanoides & . & . & . & 29 & . & . \\
\hline Climacium dendroides & $\cdot$ & $\cdot$ & $\cdot$ & $\cdot$ & 25 & $\cdot$ \\
\hline Plagiomnium cuspidatum & . & . & 55 & . & 75 & . \\
\hline Plagiomnium undulatum & . & $\cdot$ & . & . & 25 & $\cdot$ \\
\hline Brachythecium rivulare & . & . & . & . & $\cdot$ & 17 \\
\hline Leptodictyum riparium & . & . & . & . & . & 83 \\
\hline Amblystegium serpens & 8 & . & . & . & . & . \\
\hline Brachythecium reflexum & . & . & 9 & . & . & . \\
\hline Brachythecium salebrosum & 23 & 3 & 27 & 21 & $\cdot$ & $\cdot$ \\
\hline Drepanocladus aduncus & . & $\cdot$ & . & . & . & 17 \\
\hline Leskeella nervosa & 8 & 10 & 9 & $\cdot$ & $\cdot$ & $\cdot$ \\
\hline Leucodon sciuroides & . & . & . & 7 & . & . \\
\hline Lophocolea heterophylla & . & 10 & 27 & . & . & . \\
\hline Orthotrichum obtusifolium & 8 & $\cdot$ & $\cdot$ & $\cdot$ & $\cdot$ & $\cdot$ \\
\hline Plagiothecium succulentum & $\cdot$ & $\cdot$ & 18 & $\cdot$ & 25 & $\cdot$ \\
\hline Pohlia nutans & $\cdot$ & $\cdot$ & $\cdot$ & $\cdot$ & 25 & 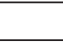 \\
\hline Ptilidium pulcherrimum & $\cdot$ & 6 & . & . & . & . \\
\hline Radula complanata & 31 & 13 & 9 & 7 & $\cdot$ & $\cdot$ \\
\hline Rhizomnium punctatum & $\cdot$ & $\cdot$ & 9 & $\cdot$ & $\cdot$ & $\cdot$ \\
\hline
\end{tabular}

Classes: FRU - Frullanio dilatatae-Leucodontetea sciuroidis, CLE - Cladonio digitatae-Lepidozietea reptantis, NEC - Neckeretea complanatae, HYL - Hylocomietea splendentis, PLA - Platyhypnidio-Fontinalietea antipyreticae.

Orders: Ort - Orthotrichetalia, Dic - Dicranetalia scoparii, Bra - Brachythecietalia rutabulo-salebrosi, Nec - Neckeretalia complanatae, Hyl - Hylocomietalia splendentis, Lep - Leptodictyetalia riparii 
non-acid bark. Character species and dominants of three associations are, respectively, Leskea polycarpa Hedw., Pylaisia polyantha (Hedw) Schimp., and Orthotrichum pumilum Sw. Available relevés were sampled on living trees of Salix alba L., Salix babylonica L., Populus canescens (Aiton) Sm., and Fraxinus excelsior.

Communities of the Dicranetalia scoparii (Tables $3,4)$ occur mainly in mesic conditions on trees with acid bark (in our dataset, on living and dead trunks of Alnus glutinosa, Betula pendula Roth, Carpinus betulus, Quercus robur, Robinia pseudoacacia L.). In the park, the order is represented by 5 associations. Character species and typical dominants of four associations are, respectively, Callicladium haldanianum (Grev.) H.A.Crum, Hypnum pallescens (Hedw.) P.Beauv., Platygyrium repens (Brid.) Schimp., and Orthodicranum montanum (Hedw.) Loeske. The association Dicrano scoparii-Hypnetum filiformis is considered to be a basal association of Dicrano scoparii-Hypnion filiformis without character and positive differental species (Marstaller, 2006). Besides 5 associations, the comm. Dicranum tauricum - [Frullanio-Leucodontetea] was included in the Dicranetalia scoparii. Dicranum tauricum Sapjegin is a character species of the predominantly epixylic class Cladonio digitatae-Lepidozietea reptantis. In the park, bryophyte communities predominated by Dicranum tauricum were found on living trees of Betula pendula and Alnus glutinosa together with the species of the class Frullanio dilatatae-Leucodontetea sciuroidis.

The Cladonio digitatae-Lepidozietea reptantis (Table 5) is represented by one order, Brachythecietalia rutabulo-salebrosi, with one alliance and two associations. It includes predominantly epixylic communities of sub-hygrophilous pleurocarpous mosses. In the park, they were sampled on dead trees of Alnus glutinosa and Quercus robur. One relevé was made on a living tree of Alnus glutinosa. The most frequent dominant is Brachythecium rutabulum (Hedw.) Schimp. One association is distinguished by the presence of Brachythecium salebrosum (Hoffm. ex F.Weber \& D.Mohr) Schimp. and Amblystegium serpens (Hedw.) Schimp.

The Neckeretea complanatae (Table 6) comprises some communities of large mat-forming sciophytic bryophytes preferring base-rich substrates. In Holosiivskyi National Nature Park, they were described on living trees of Fraxinus excelsior (most plots), Acer platanoides L., Salix alba, Quercus robur, and Tilia cordata Mill. Three identified in the park associations are characterised respectively by Anomodon attenuatus
(Hedw.) Huebener, A. longifolius (Schleich. ex Brid.) Hartm., and Homalia trichomanoides (Hedw.) Schimp. In addition, an unidentified community with dominance of Anomodon viticulosus (Hedw.) Hook. \& Taylor was found. Communities of this class are rarer in the park than those of the classes Frullanio dilatatae-Leucodontetea sciuroidis and Cladonio digitatae-Lepidozietea reptantis.

The Platyhypnidio-Fontinalietea antipyreticae (Table 7) is a class of hydrophilous bryophyte vegetation occurring in and near fresh waters. Relevés of this class were sampled on dead trunks and branches of Alnus glutinosa lying in floodplains of rivulets. Height above the ground was from 10 to $45 \mathrm{~cm}$. The class is represented by two associations; both contain only species-poor variants. Their character and main dominant species are respectively Leptodictyum riparium (Hedw.) Warnst. and Brachythecium rivulare Schimp. Relevés with Leptodictyum riparium were identified as the association Leptodictyo riparii-Hygroamblystegietum varii (Hugonnot, Celle, 2013). Our relevés of this association do not include the second character species Hygroamblystegium varium (Hedw.) Monk.; however, this species occurs in the park in the same habitats. The authors of the association placed it in the Cladonio digitatae-Lepidozietea reptantis. Taking into account that the association is confined to wet habitats and Leptodictyum riparium is a character species of the class Platyhypnidio-Fontinalietea antipyreticae (Marstaller, 2006), the association may belong to this class.

The Hylcomietea splendentis (Table 7) is a class of predominantly epigeic bryophyte communities. In the park, they are described on dead logs of Alnus glutinosa and Quercus robur at the late decay stage.

In Holosiivskyi National Nature Park, most frequent dominants of bryophyte communities are Hypnum cupressiforme and $H$. pallescens on living trees and Brachythecium rutabulum on dead wood. Trees in mesic oak-hornbeam forests usually do not have bryophyte communities. Much more often, they occur in wet forests, near water, and in mesic forests in ravines.

Associations Callicladietum haldaniani LeBlanc 1963, Brachythecio rutabuli-Plagiomnietum cuspidati (Felföldy 1941) Plamada 1982, Brachythecio rivularisHygrohypnetum luridi Philippi 1965, and Leptodictyo riparii-Hygroamblystegietum varii Hugonnot et Celle 2013 are reported from Ukraine for the first time. The latter two associations are represented by the impoverished variants without a part of their diagnostic species. 
Table 2. Relevés of the order Orthotrichetalia Hadač in Klika et Hadač 1944

\begin{tabular}{|c|c|c|c|c|c|c|c|c|c|c|c|c|c|}
\hline Number in table & 1 & 2 & 3 & 4 & 5 & 6 & 7 & 8 & 9 & 10 & 11 & 12 & 13 \\
\hline $\begin{array}{l}\text { Number in database of Holosiivskyi } \\
\text { NNP }\end{array}$ & 1517 & 1513 & 1514 & 1567 & 1502 & 1580 & 1576 & 1515 & 1579 & 1504 & 1537 & 1583 & 1503 \\
\hline Number in database EU-UA-006 & 3878 & 3874 & 3875 & 3928 & 3863 & 3941 & 3937 & 3876 & 3940 & 3865 & 3898 & 3944 & 3864 \\
\hline Area $\left(\mathrm{dm}^{2}\right)$ & 4 & 4 & 4 & 4 & 4 & 4 & 4 & 4 & 4 & 4 & 4 & 4 & 4 \\
\hline Aspect & NWW & SW & $\mathrm{NE}$ & SWW & S & W & NEE & NNW & W & SW & SSE & W & SW \\
\hline Number of bryophyte species & 1 & 2 & 2 & 2 & 2 & 5 & 6 & 2 & 5 & 3 & 5 & 2 & 4 \\
\hline Living tree & $*$ & $*$ & $*$ & $*$ & $*$ & $*$ & $*$ & $*$ & $*$ & $*$ & $*$ & $*$ & $*$ \\
\hline Tree species & $\mathrm{Cb}$ & Pca & Pca & Fex & Sba & Pca & Cavi & Apl & $?$ & Sba & $\mathrm{Cb}$ & $\mathrm{Sa}$ & Sba \\
\hline Height above the ground $(\mathrm{cm})$ & 25 & 170 & 170 & 50 & 140 & 180 & 140 & 60 & 160 & 190 & 110 & 220 & 180 \\
\hline \multicolumn{14}{|l|}{ Ch Frullanio-Leucodontetea } \\
\hline Leskea polycarpa & 85 & 63 & 74 & 75 & 49 & 80 & 60 & 37 & 80 & 10 & . & . & 10 \\
\hline Pylaisiella polyantha & . & . & . & . & & 3 & 10 & 18 & 3 & 37 & 48 & 70 & 10 \\
\hline Orthotrichum pumilum & . & . & . & . & 1 & 5 & 8 & . & 5 & 3 & . & . & 20 \\
\hline Orthotrichum speciosum & . & . & . & . & . & . & 7 & . & . & . & + & 20 & . \\
\hline Orthotrichum obtusifolium & . & . & . & . & . & . & . & . & . & . & . & . & + \\
\hline \multicolumn{14}{|l|}{ Ch Cladonio-Lepidozietea } \\
\hline Amblystegium serpens & . & . & . & . & . & . & 5 & . & $\cdot$ & . & . & . & . \\
\hline Bryum moravicum & . & . & . & . & . & . & . & . & . & . & + & . & . \\
\hline Orthotrichum sp. & . & . & . & . & . & . & + & . & . & . & . & . & $\cdot$ \\
\hline
\end{tabular}

Associations: 1 - Leskeetum polycarpae; 2 - Pylaisietum polyanthae; 3 - Orthotrichetum pumili.

Abbreviations of tree names: Agl - Alnus glutinosa, Cavi - Cerasus avium, $\mathrm{Cb}-$ Carpinus betulus, Fex - Fraxinus excelsior, Pca Populus $\times$ canescens, $\mathrm{Qr}-$ Quercus robur, $\mathrm{Sa}-$ Salix alba, Sba - Salix babylonica.

Dates and locations:

1517 - 2017.04.28, Maksym Rylskyi Holosiivskyi Park, latitude 50.38911 ${ }^{\circ}$, longitude $30.50541^{\circ}$, accuracy 5 m; 1513 - 2017.04.28, Maksym Rylskyi Holosiivskyi Park, latitude 50.39222 ${ }^{\circ}$, longitude $30.51064^{\circ}$, accuracy 8 m; 1514 - 2017.04.28, Maksym Rylskyi Holosiivskyi Park, latitude 50.39222 ${ }^{\circ}$, longitude $30.51064^{\circ}$, accuracy 8 m; 1567 - 2017.05.02, Lisnyky reserve, latitude 50.29599 , longitude $30.53662^{\circ}$, accuracy $5 \mathrm{~m}$;

1502 - 2017.04.28, Maksym Rylskyi Holosiivskyi Park, latitude 50.39361 ${ }^{\circ}$, longitude $30.51118^{\circ}$, accuracy 9 m; 1580 - 2018.04.26, Teremky, latitude $50.36022^{\circ}$, longitude $30.44494^{\circ}$, accuracy $6 \mathrm{~m}$;

1576 - 2018.04.26, Teremky, latitude $50.36013^{\circ}$, longitude $30.44732^{\circ}$, accuracy $6 \mathrm{~m}$;

1515 - 2017.04.28, Maksym Rylskyi Holosiivskyi Park, latitude 50.39110 , longitude $30.51017^{\circ}$, accuracy 8 m; 1579 - 2018.04.26, Teremky, latitude $50.35863^{\circ}$, longitude $30.44836^{\circ}$, accuracy $6 \mathrm{~m}$;

1504 - 2017.04.28, Maksym Rylskyi Holosiivskyi Park, latitude 50.39361 ${ }^{\circ}$, longitude $30.51118^{\circ}$, accuracy $9 \mathrm{~m}$; 1537 - 2017.04.30, Holosiivskyi Wood, latitude 50.37232 ${ }^{\circ}$, longitude $30.49988^{\circ}$, accuracy $8 \mathrm{~m}$; 1583 - 2018.05.05, Sviatoshynske forestry, latitude $50.49061^{\circ}$, longitude $30.31212^{\circ}$, accuracy $6 \mathrm{~m}$;

1503 - 2017.04.28, Maksym Rylskyi Holosiivskyi Park, latitude 50.39361 ${ }^{\circ}$, longitude $30.51118^{\circ}$, accuracy 9 m. 
Table 3. Relevés of the order Dicranetalia scopariae Barkman 1958 (associations Platygyrietum repentis Leblanc 1963, Callicladietum haldaniani Leblanc 1963), com. Dicranum tauricum - [Frullanio-Leucodontetea]

\begin{tabular}{|c|c|c|c|c|c|c|c|c|c|c|c|c|c|}
\hline Number in table & 1 & 2 & 3 & 4 & 5 & 6 & 7 & 8 & 9 & 10 & 11 & 12 & 13 \\
\hline $\begin{array}{l}\text { Number in database of Holosiivskyi } \\
\text { NNP }\end{array}$ & 1505 & 1506 & 1511 & 1547 & 1520 & 1550 & 1561 & 1590 & 1563 & 1512 & 1573 & 1574 & 1584 \\
\hline Number in database EU-UA-006 & 3866 & 3867 & 3872 & 3908 & 3881 & 3911 & 3922 & 3951 & 3924 & 3873 & 3934 & 3935 & 3945 \\
\hline Area $\left(\mathrm{dm}^{2}\right)$ & 4 & 4 & 4 & 4 & 4 & 4 & 4 & 4 & 4 & 4 & 4 & 4 & 4 \\
\hline Aspect & SWW & SW & SE & $\mathrm{W}$ & SWW & - & NEE & NW & SEE & NWW & NW & NW & SW \\
\hline Inclination $\left(^{\circ}\right)$ & 60 & 70 & 60 & 80 & 80 & 0 & 78 & 77 & 70 & 80 & 70 & 70 & 70 \\
\hline Cover of bryophytes (\%) & 60 & 40 & 80 & 60 & 50 & 70 & 65 & 45 & 70 & 75 & 90 & 95 & 35 \\
\hline Number of bryophyte species & 1 & 2 & 4 & 3 & 1 & 5 & 3 & 2 & 2 & 1 & 5 & 5 & 2 \\
\hline Living tree & $*$ & * & - & $*$ & $*$ & - & * & * & - & - & $*$ & $*$ & * \\
\hline Tree species & Qr & Agl & Agl & Qr & Qr & Fex & $\mathrm{Bp}$ & $\mathrm{Agl}$ & Qr & Agl & $\mathrm{Bp}$ & $\mathrm{Bp}$ & Agl \\
\hline Height above the ground $(\mathrm{cm})$ & 15 & 130 & 60 & 100 & 10 & 60 & 170 & 130 & 50 & 35 & 60 & 90 & 160 \\
\hline Association & \multicolumn{8}{|c|}{1} & \multicolumn{2}{|c|}{2} & \multicolumn{3}{|c|}{3} \\
\hline \multicolumn{14}{|l|}{ Ch Frullanio-Leucodontetea } \\
\hline Platygyrium repens & 60 & 40 & 70 & 55 & 50 & 10 & 60 & 40 & . & . & . & 1 & . \\
\hline Callicladium haldanianum & . & . & . & . & . & . & . & . & 70 & 75 & . & . & . \\
\hline Radula complanata & . & . & . & 5 & . & 5 & . & . & . & . & . & . & . \\
\hline Hypnum cupressiforme & . & . & + & . & . & 40 & & . & . & . & . & . & $\cdot$ \\
\hline Hypnum pallescens & . & + & + & . & . & . & 5 & . & . & . & . & 1 & 5 \\
\hline Lophocolea heterophylla & . & . & . & . & . & . & . & . & . & . & 3 & . & . \\
\hline Orthodicranum montanum & . & . & + & . & . & . & + & 5 & . & . & 1 & 3 & $\cdot$ \\
\hline Pylaisiella polyantha & . & . & . & . & . & 10 & . & . & . & . & . & . & . \\
\hline \multicolumn{14}{|l|}{ Other species } \\
\hline Climacium dendroides & . & . & . & . & . & . & . & . & + & . & . & . & . \\
\hline Dicranum tauricum & . & . & . & . & . & . & . & . & . & . & 80 & 80 & 30 \\
\hline Dicranum scoparium & . & . & . & . & . & . & . & . & . & . & 1 & . & . \\
\hline Leskeela nervosa & . & . & . & + & . & . & . & . & . & . & . & . & . \\
\hline Orthotrichum speciosum & . & . & . & . & . & 5 & . & . & . & . & . & . & $\cdot$ \\
\hline Ptilidium pulcherrimum & . & . & . & . & . & . & . & . & . & . & 5 & 5 & . \\
\hline
\end{tabular}

Associations: 1 - Platygyrietum repentis; 2 - Callicladietum haldaniani; 3 - com. Dicranum tauricum - [Frullanio-Leucodontetea $]$. Abbreviations of tree names: Agl - Alnus glutinosa, Bp - Betula pendula, Fex - Fraxinus excelsior, Qr - Quercus robur.

Dates and locations:

1505 - 2017.04.28, Maksym Rylskyi Holosiivskyi Park, latitude 50.39382 ${ }^{\circ}$, longitude $30.51229^{\circ}$, accuracy 8 m;

1506 - 2017.04.28, Maksym Rylskyi Holosiivskyi Park, latitude 50.39316 ${ }^{\circ}$, longitude $30.51200^{\circ}$, accuracy $8 \mathrm{~m}$;

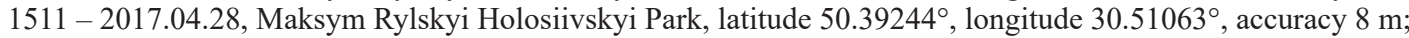

1547 - 2017.04.30, Lisnyky reserve, latitude $50.29635^{\circ}$, longitude $30.53629^{\circ}$, accuracy $5 \mathrm{~m}$;

1520 - 2017.04.28, Maksym Rylskyi Holosiivskyi Park, latitude 50.39020 , longitude $30.50330^{\circ}$, accuracy $5 \mathrm{~m}$;

1550 - 2017.05.02, Lisnyky reserve, latitude $50.29620^{\circ}$, longitude $30.53612^{\circ}$, accuracy $4 \mathrm{~m}$;

1561 - 2017.05.02, Lisnyky reserve, latitude $50.29702^{\circ}$, longitude $30.54237^{\circ}$, accuracy $6 \mathrm{~m}$;

$1590-2018.05 .05$, Sviatoshynske forestry, latitude $50.49115^{\circ}$, longitude $30.31044^{\circ}$, accuracy $6 \mathrm{~m}$;

1565 - 2017.05.02, Lisnyky reserve, latitude $50.29658^{\circ}$, longitude $30.54676^{\circ}$, accuracy $7 \mathrm{~m}$;

1512 - 2017.04.28, Maksym Rylskyi Holosiivskyi Park, latitude 50.39244 ${ }^{\circ}$, longitude $30.51063^{\circ}$, accuracy 8 m;

1573 - 2018.04.25, Dachne forestry, latitude $50.25709^{\circ}$, longitude $30.60036^{\circ}$, accuracy $6 \mathrm{~m}$;

1574 - 2018.04.25, Dachne forestry, latitude $50.25709^{\circ}$, longitude $30.60036^{\circ}$, accuracy $6 \mathrm{~m}$;

$1584-2018.05 .05$, Sviatoshynske forestry, latitude $50.490585^{\circ}$, longitude $30.31132^{\circ}$, accuracy $6 \mathrm{~m}$. 
Table 4. Relevés of the order Dicranetalia scopariae Barkman 1958 (associations Ptilidio pulcherrimi-Hypnetum pallescentis Herzog 1943, Dicrano montani-Hypnetum cupressiformis Wiśniewski 1930, Dicrano scoparii-Hypnetum filiformis Barkman 1949)

\begin{tabular}{|c|c|c|c|c|c|c|c|c|c|c|c|c|c|c|c|c|c|c|c|}
\hline Number in table & 1 & 2 & 3 & 4 & 5 & 6 & 7 & 8 & 9 & 10 & 11 & 12 & 13 & 14 & 15 & 16 & 17 & 18 & 19 \\
\hline $\begin{array}{l}\text { Number in database of Holosiivskyi } \\
\text { NNP }\end{array}$ & $\frac{n}{n}$ & $\stackrel{n}{n}$ & $\stackrel{\circ}{\underline{n}}$ & $\stackrel{\infty}{i}$ & $\underset{n}{\mathbb{n}}$ & $\begin{array}{l}8 \\
\stackrel{0}{n}\end{array}$ & $\stackrel{a}{\underline{n}}$ & $\stackrel{\infty}{n}$ & $\begin{array}{l}\vec{\infty} \\
\stackrel{n}{n}\end{array}$ & $\begin{array}{l}\infty \\
\infty \\
n\end{array}$ & $\stackrel{\substack{n \\
n}}{n}$ & $\underset{⿱}{ \pm}$ & 点 & กิ & $\overrightarrow{\tilde{n}}$ & $\vec{\circ}$ & $\stackrel{n}{\hat{2}}$ & $\stackrel{2}{\circ}$ & $\stackrel{\infty}{n}$ \\
\hline Number in database EU-UA-006 & in & 市 & $\underset{\substack{\infty \\
m}}{\vec{n}}$ & ळे & $\hat{m}$ & $\overrightarrow{\widehat{N}}$ & $\begin{array}{l}\infty \\
\infty \\
\infty \\
\infty\end{array}$ & ஜ̊ & ॠั & के & $\vec{\infty}$ & 年 & ๙ิ & $\underset{\infty}{\infty}$ & $\underset{\infty}{\sim}$ & 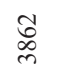 & $\underset{\substack{\infty \\
\infty}}{\infty}$ & 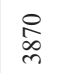 & 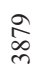 \\
\hline Area $\left(\mathrm{dm}^{2}\right)$ & 4 & 4 & 4 & 4 & 4 & 4 & 4 & 4 & 4 & 4 & 4 & 4 & 4 & 4 & 4 & 4 & 4 & 4 & 4 \\
\hline Aspect & 3 & 3 & 넹 & $\sum_{z}^{3}$ & a. & 四 & 之 & 之 & 之 & z & z & 党 & 3 & 3 & in & $\sum_{z}^{3}$ & $\sum_{z}^{3}$ & ' & $\sum_{z}$ \\
\hline Inclination $\left({ }^{\circ}\right)$ & 65 & 80 & 45 & 75 & 75 & 65 & 50 & 65 & 70 & 70 & 65 & 70 & 80 & 22 & 45 & 75 & 55 & 0 & 85 \\
\hline Cover of bryophytes (\%) & 70 & 90 & 80 & 90 & 55 & 85 & 90 & 70 & 80 & 55 & 85 & 35 & 90 & 85 & 85 & 65 & 50 & 90 & 90 \\
\hline Number of bryophyte species & 4 & 2 & 1 & 3 & 2 & 2 & 3 & 3 & 2 & 2 & 4 & 2 & 2 & 3 & 3 & 2 & 1 & 1 & 1 \\
\hline Living tree & $*$ & $*$ & - & $*$ & $*$ & $*$ & $*$ & $*$ & $*$ & $*$ & - & $*$ & $*$ & - & - & $*$ & $*$ & - & $*$ \\
\hline Tree species & $\mathrm{Bp}$ & $\mathrm{Agl}$ & $\mathrm{Agl}$ & $\mathrm{Bp}$ & $\mathrm{Bp}$ & $\mathrm{Bp}$ & $\mathrm{Qr}$ & $\mathrm{Agl}$ & Qr & $\mathrm{Agl}$ & $\mathrm{Qr}$ & $\mathrm{Agl}$ & $\mathrm{Bp}$ & $\mathrm{Qr}$ & $\mathrm{Qr}$ & Rps & $\mathrm{Agl}$ & $\mathrm{Agl}$ & $\mathrm{Cb}$ \\
\hline Height above the ground $(\mathrm{cm})$ & 140 & 40 & 60 & 25 & 110 & 120 & 10 & 120 & 40 & 120 & 20 & 150 & 100 & $?$ & 110 & 130 & 110 & 60 & 30 \\
\hline Association & \multicolumn{9}{|c|}{7} & \multicolumn{4}{|c|}{2} & \multicolumn{6}{|c|}{3} \\
\hline \multicolumn{20}{|l|}{$\begin{array}{l}\text { Ch Frullanio dilatatae- } \\
\text { Leucodontetea sciuroidis }\end{array}$} \\
\hline Hypnum pallescens & 60 & 90 & 80 & 68 & 54 & 80 & 30 & 60 & 70 & 5 & . & 5 & 5 & . & . & . & . & . & . \\
\hline Orthodicranum montanum & 2 & . & . & . & . & . & . & 5 & 10 & 50 & 55 & 30 & 85 & . & . & . & . & . & . \\
\hline Hypnum cupressiforme & . & . & . & . & . & 5 & 60 & 5 & . & . & 28 & . & . & 70 & 60 & 60 & 50 & 90 & 90 \\
\hline Dicranum tauricum & . & . & . & 20 & . & . & . & . & . & . & . & . & . & . & . & $\cdot$ & . & . & $\cdot$ \\
\hline Leskeella nervosa & . & . & . & . & . & . & . & . & . & . & . & . & . & 15 & 20 & . & . & . & $\cdot$ \\
\hline Platygyrium repens & 5 & + & . & . & . & . & . & . & . & . & . & . & . & $\cdot$ & . & 5 & . & . & . \\
\hline Radula complanata & $\cdot$ & . & · & . & 1 & . & . & . & . & $\cdot$ & . & . & . & · & + & · & . & . & $\cdot$ \\
\hline \multicolumn{20}{|l|}{ Ch Cladonio-Lepidozietea } \\
\hline Brachythecium salebrosum & . & . & . & . & . & . & . & . & . & . & . & . & . & + & . & . & . & . & . \\
\hline Dicranum flagellare & 3 & . & . & . & . & . & . & . & . & . & . & . & . & $\cdot$ & . & . & . & . & . \\
\hline Dicranum viride & . & · & . & . & . & . & . & · & . & . & + & . & . & . & . & . & . & . & . \\
\hline \multicolumn{19}{|l|}{ Other species } & . \\
\hline Bryum moravicum & . & . & . & . & . & . & + & . & . & . & . & . & . & . & . & . & . & . & . \\
\hline
\end{tabular}

Associations: 1 - Ptilidio pulcherrimi-Hypnetum pallescentis; 2 - Dicrano montani-Hypnetum cupressiformi; 3 - Dicrano scopariiHypnetum filiformis.

Abbreviations of tree names: Agl - Alnus glutinosa, Bp - Betula pendula, $\mathrm{Cb}$-Carpinus betulus, Qr - Quercus robur, Rps - Robinia pseudoacacia.

Dates and locations:

1575 - 2018.04.25, Dachne forestry, latitude 50.25323, longitude $30.60261^{\circ}$, accuracy $6 \mathrm{~m}$;

1587 - 2018.05.05, Sviatoshynske forestry, latitude $50.49108^{\circ}$, longitude $30.31033^{\circ}$, accuracy $6 \mathrm{~m}$;

1510 - 2017.04.28, Maksym Rylskyi Holosiivskyi Park, latitude 50.39258 ${ }^{\circ}$, longitude $30.51060^{\circ}$, accuracy $11 \mathrm{~m}$;

1578 - 2018.04.26, Teremky, latitude $50.35712^{\circ}$, longitude $30.44472^{\circ}$, accuracy $6 \mathrm{~m}$;

1573 - 2018.04.25, Dachne forestry, latitude $50.25709^{\circ}$, longitude $30.60036^{\circ}$, accuracy $6 \mathrm{~m}$;

1560 - 2017.05.02, Lisnyky reserve, latitude $50.29702^{\circ}$, longitude $30.54237^{\circ}$, accuracy $6 \mathrm{~m}$;

1519 - 2017.04.28, Maksym Rylskyi Holosiivskyi Park, latitude 50.38839 ${ }^{\circ}$, longitude $30.50451^{\circ}$, accuracy $8 \mathrm{~m}$;

1589 - 2018.05.05, Sviatoshynske forestry, latitude $50.49108^{\circ}$, longitude $30.31033^{\circ}$, accuracy $10 \mathrm{~m}$;

1581 - 2018.05.05, Sviatoshynske forestry, latitude $50.48955^{\circ}$, longitude $30.31427^{\circ}$, accuracy $6 \mathrm{~m}$;

1574 - 2018.04.25, Dachne forestry, latitude $50.25709^{\circ}$, longitude $30.60036^{\circ}$, accuracy $6 \mathrm{~m}$;

1588 - 2018.05.05, Sviatoshynske forestry, latitude $50.49108^{\circ}$, longitude $30.310328^{\circ}$, accuracy $6 \mathrm{~m}$;

1530 - 2017.04.30, Holosiivskyi Wood, latitude $50.37303^{\circ}$, longitude $30.50025^{\circ}$, accuracy $8 \mathrm{~m}$;

1584 - 2018.05.05, Sviatoshynske forestry, latitude $50.490585^{\circ}$, longitude $30.31132^{\circ}$, accuracy $6 \mathrm{~m}$;

1541 - 2017.04.30, Holosiivskyi Wood, latitude $50.37177^{\circ}$, longitude $30.49885^{\circ}$, accuracy $6 \mathrm{~m}$;

1529 - 2017.04.30, Holosiivskyi Wood, latitude $50.37335^{\circ}$, longitude $30.499780^{\circ}$, accuracy $9 \mathrm{~m}$;

1531 - 2017.04.30, Holosiivskyi Wood, latitude 50.37309 ${ }^{\circ}$, longitude $30.50000^{\circ}$, accuracy $7 \mathrm{~m}$;

1501 - 2017.04.28, Maksym Rylskyi Holosiivskyi Park, latitude 50.39351 ${ }^{\circ}$, longitude $30.51041^{\circ}$, accuracy $8 \mathrm{~m}$; 1507 - 2017.04.28, Maksym Rylskyi Holosiivskyi Park, latitude 50.39316 ${ }^{\circ}$, longitude $30.51200^{\circ}$, accuracy $8 \mathrm{~m}$; 1509 - 2017.04.28, Maksym Rylskyi Holosiivskyi Park, latitude 50.37275 ${ }^{\circ}$, longitude $30.51092^{\circ}$, accuracy 8 m; 1518 - 2017.04.28, Maksym Rylskyi Holosiivskyi Park, latitude 50.38853 ${ }^{\circ}$, longitude $30.50478^{\circ}$, accuracy 6 m. 
Table 5. Relevés of the order Brachythecietalia rutabulo-salebrosi Marstaller 1987

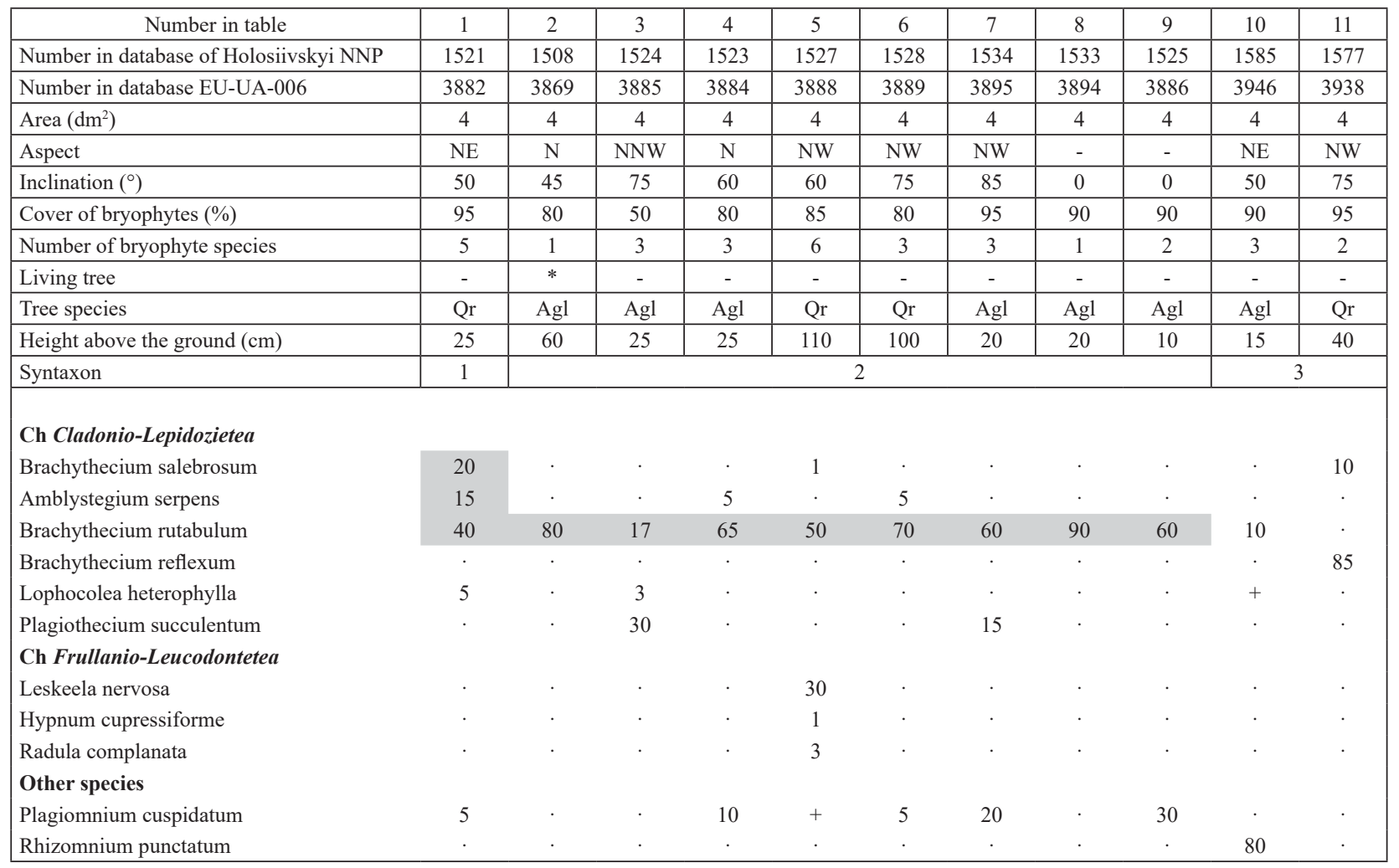

Syntaxa: 1 - Brachythecio salebrosi-Amblystegietum juratzkani; 2 - Brachythecio rutabuli-Hypnetum cupressiformis; 3 - Bryo capillaris-Brachythecion rutabuli, association is not identified.

Abbreviations of tree names: Agl - Alnus glutinosa, Qr - Quercus robur.

Dates and locations:

1521 - 2017.04.30, Holosiyivskyi Wood, latitude 50.37441 ${ }^{\circ}$, longitude $30.50001^{\circ}$, accuracy $6 \mathrm{~m}$;

1508 - 2017.04.28, Maksym Rylskyi Holosiivskyi Park, latitude 50.39275², longitude $30.51092^{\circ}$, accuracy $8 \mathrm{~m}$;

1524 - 2017.04.30, Holosiyivskyi Wood, latitude $50.37315^{\circ}$, longitude $30.49993^{\circ}$, accuracy $11 \mathrm{~m}$;

1523 - 2017.04.30, Holosiyivskyi Wood, latitude $50.37343^{\circ}$, longitude $30.49963^{\circ}$, accuracy $10 \mathrm{~m}$;

1527 - 2017.04.30, Holosiyivskyi Wood, latitude $50.37335^{\circ}$, longitude $30.49978^{\circ}$, accuracy $9 \mathrm{~m}$;

1528 - 2017.04.30, Holosiyivskyi Wood, latitude $50.37335^{\circ}$, longitude $30.49978^{\circ}$, accuracy $9 \mathrm{~m}$;

1534 - 2017.04.30, Holosiyivskyi Wood, latitude 50.37282 ${ }^{\circ}$, longitude $30.49983^{\circ}$, accuracy $9 \mathrm{~m}$;

1533 - 2017.04.30, Holosiyivskyi Wood, latitude $50.37282^{\circ}$, longitude $30.49993^{\circ}$, accuracy $9 \mathrm{~m}$;

1525 - 2017.04.30, Holosiyivskyi Wood, latitude $50.37361^{\circ}$, longitude $30.49975^{\circ}$, accuracy $10 \mathrm{~m}$;

1585 - 2017.05.05, Sviatoshynske forestry, latitude $50.37315^{\circ}$, longitude $30.31111^{\circ}$, accuracy $6 \mathrm{~m}$;

1577 - 2017.04.26, Teremky, latitude $50.35773^{\circ}$, longitude $30.44586^{\circ}$, accuracy $6 \mathrm{~m}$. 
Table 6. Relevés of the class Neckeretea complanatae Marstaller 1986

\begin{tabular}{|c|c|c|c|c|c|c|c|c|c|c|c|c|c|}
\hline Number in table & 1 & 2 & 3 & 5 & 6 & 7 & 8 & 9 & 10 & 11 & 12 & 13 & 14 \\
\hline $\begin{array}{l}\text { Number in database of Holosiivskyi } \\
\text { NNP }\end{array}$ & 1549 & 1566 & 1571 & 1570 & 1565 & 1538 & 1539 & 1552 & 1564 & 1551 & 1546 & 1548 & 1553 \\
\hline Number in database EU-UA-006 & 3910 & 3927 & 3932 & 3931 & 3926 & 3899 & 3900 & 3913 & 3925 & 3912 & 3907 & 3909 & 3914 \\
\hline Aspect & SW & SWW & - & $\mathrm{W}$ & $\mathrm{NE}$ & $\mathrm{E}$ & SSW & NNW & SE & NNW & NNE & $\mathrm{NE}$ & NWW \\
\hline Inclination $\left(^{\circ}\right)$ & 70 & 75 & 0 & 55 & 75 & 70 & 80 & 70 & 60 & 70 & 70 & 70 & 70 \\
\hline Cover of bryophytes (\%) & 95 & 100 & 100 & 100 & 60 & 95 & 70 & 80 & 90 & 95 & 95 & 95 & 50 \\
\hline Living tree & $*$ & $*$ & $*$ & $*$ & $*$ & $*$ & $*$ & $*$ & $*$ & $*$ & $*$ & $*$ & $*$ \\
\hline Tree species & Fex & Fex & Fex & Fex & Tco & Apl & Apl & Sal & Fex & Fex & Qr & Fex & Sal \\
\hline Height above the ground $(\mathrm{cm})$ & 20 & 110 & 60 & 40 & 170 & 100 & 40 & 40 & 35 & 20 & 10 & 20 & 70 \\
\hline Syntaxon & \multicolumn{6}{|c|}{1} & \multicolumn{2}{|c|}{2} & \multicolumn{2}{|c|}{3} & \multicolumn{2}{|c|}{4} & 5 \\
\hline \multicolumn{14}{|l|}{ Ch Neckeretea complanatae } \\
\hline Anomodon viticulosus & & 80 & 15 & 5 & . & 10 & 5 & . & . & . & . & . & 40 \\
\hline Amblystegium subtile & 3 & . & . & . & . & . & . & . & . & 5 & . & 5 & . \\
\hline \multicolumn{14}{|l|}{ Ch Frullanio-Leucodontetea } \\
\hline Leucodon sciuroides & . & . & 25 & . & . & . & . & . & . & . & . & . & . \\
\hline Hypnum cupressiforme & . & . & . & . & . & . & . & . & . & . & . & . & 5 \\
\hline Radula complanata & . & . & . & . & . & . & . & + & . & . & . & . & . \\
\hline \multicolumn{14}{|l|}{ Ch Cladonio-Lepidozietea } \\
\hline Brachythecium salebrosum & 2 & . & . & . & . & . & . & 5 & . & . & . & . & 5 \\
\hline
\end{tabular}

Syntaxa: 1 - Anomodontetum attenuati; 2 - Anomodontetum longifolii; 3 - transition Anomodontetum longifolii $\times$ Plagiomnio cuspidati-Homalietum trichomanoidis; 4 - Plagiomnio cuspidati-Homalietum trichomanoidis; 5 - Neckerion complanatae, association is not identified.

Abbreviations of tree names: Apl - Acer platanoides, Fex - Fraxinus excelsior, Qr - Quercus robur, Sal - Salix alba, Tco - Tilia cordata

Dates and locations:

1549 - 2017.05.02, Lisnyky, latitude $50.29638^{\circ}$, longitude $30.53635^{\circ}$, accuracy $7 \mathrm{~m}$;

1566 - 2017.05.02, Lisnyky, latitude $50.29618^{\circ}$, longitude $30.54686^{\circ}$, accuracy $13 \mathrm{~m}$;

1571 - 2017.05.02, Lisnyky, latitude $50.29502^{\circ}$, longitude $30.55236^{\circ}$, accuracy $7 \mathrm{~m}$; 1570 - 2017.05.02, Lisnyky, latitude $50.29608^{\circ}$, longitude $30.54687^{\circ}$, accuracy $7 \mathrm{~m}$; 1568 - 2017.05.02, Lisnyky, latitude $50.29618^{\circ}$, longitude $30.54686^{\circ}$, accuracy $13 \mathrm{~m}$; 1565 - 2017.05.02, Lisnyky, latitude $50.29658^{\circ}$, longitude $30.54678^{\circ}$, accuracy $7 \mathrm{~m}$; 1538 - 2017.04.30, Holosiyivskyi Wood, latitude $50.37228^{\circ}$, longitude $30.49991^{\circ}$, accuracy $6 \mathrm{~m}$; 1539 - 2017.04.30, Holosiyivskyi Wood, latitude 50.37228 , longitude $30.49991^{\circ}$, accuracy $6 \mathrm{~m}$; 1552 - 2017.05.02, Lisnyky, latitude $50.29575^{\circ}$, longitude $30.53789^{\circ}$, accuracy $7 \mathrm{~m}$; 1564 - 2017.05.02, Lisnyky, latitude $50.29660^{\circ}$, longitude $30.54680^{\circ}$, accuracy $6 \mathrm{~m}$; 1551 - 2017.05.02, Lisnyky, latitude $50.29576^{\circ}$, longitude $30.53750^{\circ}$, accuracy $6 \mathrm{~m}$; 1546 - 2017.05.02, Lisnyky, latitude $50.29635^{\circ}$, longitude $30.53629^{\circ}$, accuracy $5 \mathrm{~m}$; 1548 - 2017.05.02, Lisnyky, latitude $50.29638^{\circ}$, longitude $30.53632^{\circ}$, accuracy $5 \mathrm{~m}$; 1553 - 2017.05.02, Lisnyky, latitude $50.29575^{\circ}$, longitude $30.53789^{\circ}$, accuracy $6 \mathrm{~m}$. 
Table 7. Relevés of the classes Platyhypnidio-Fontinalietea antipyreticae and Hylocomietea splendentis

\begin{tabular}{|c|c|c|c|c|c|c|c|c|c|c|}
\hline Number in table & 1 & 2 & 3 & 4 & 5 & 6 & 7 & 8 & 9 & 10 \\
\hline Number in database of Holosiivskyi NNP & 1526 & 1522 & 1536 & 1562 & 1555 & 1559 & 1558 & 1556 & 1586 & 1532 \\
\hline Number in database EU-UA-006 & 3887 & 3883 & 3897 & 3923 & 3916 & 3920 & 3919 & 3917 & 3947 & 3893 \\
\hline Area $\left(\mathrm{dm}^{2}\right)$ & 4 & 4 & 4 & 4 & 4 & 4 & 4 & 4 & 4 & 4 \\
\hline Inclination & 0 & 0 & 40 & 40 & 0 & 0 & 0 & 0 & 10 & 0 \\
\hline Cover of bryophytes (\%) & 100 & 100 & 80 & 80 & 90 & 100 & 90 & 90 & - & 90 \\
\hline Number of bryophyte species & 2 & 1 & 3 & 2 & 3 & 1 & 2 & 1 & 1 & 1 \\
\hline Tree species & Agl & $?$ & Agl & Qr & $\mathrm{Agl}$ & $\mathrm{Agl}$ & $\mathrm{Agl}$ & $\mathrm{Agl}$ & Agl & $\mathrm{Agl}$ \\
\hline Height above the ground $(\mathrm{cm})$ & 25 & 10 & 30 & 50 & 15 & 15 & 25 & 20 & 45 & 10 \\
\hline Height above the water $(\mathrm{cm})$ & - & - & - & - & - & 7 & 10 & 10 & 25 & 7 \\
\hline Syntaxon & \multicolumn{3}{|c|}{1} & 2 & \multicolumn{5}{|c|}{3} & 4 \\
\hline \multicolumn{11}{|l|}{ Ch Hylocomietea splendentis } \\
\hline \multicolumn{11}{|l|}{ Ch Platyhypnidio-Fontinalietea } \\
\hline Leptodictyum riparium & . & . & . & . & 80 & 100 & 15 & 95 & 95 & . \\
\hline Brachythecium rivulare & . & . & $\cdot$ & . & . & . & . & . & $\cdot$ & 90 \\
\hline \multicolumn{11}{|l|}{ Ch Cladonio-Lepidozietea } \\
\hline Brachythecium rutabulum & 15 & . & $\cdot$ & . & 5 & . & . & . & . & . \\
\hline Pohlia nutans & . & . & · & 30 & . & · & . & $\cdot$ & . & . \\
\hline Plagiothecium succulentum & . & . & 5 & . & . & . & . & . & . & . \\
\hline \multicolumn{11}{|l|}{ Other species } \\
\hline Drepanocladus aduncus & . & . & . & . & . & . & 75 & . & . & . \\
\hline
\end{tabular}

Syntaxa: 1 - Brachythecio rutabuli-Plagiomnietum cuspidati; 2 -Climacion dendroidis, association is not identified; 3 - Leptodictyo riparii-Hygroamblystegietum varii; 4 - Brachythecio rivularis-Hygrohypnetum luridi.

Abbreviations of tree names: Agl - Alnus glutinosa, Qr - Quercus robur.

Dates and locations:

1549 - 2017.05.02, Lisnyky, latitude $50.29638^{\circ}$, longitude $30.53635^{\circ}$, accuracy $7 \mathrm{~m}$;

1522 - 2017.04.30, Holosiivskyi wood, latitude $50.37330^{\circ}$, longitude $30.49960^{\circ}$, accuracy $14 \mathrm{~m}$;

1536 - 2017. 04.30, Holosiivskyi wood, latitude $50.37263^{\circ}$, longitude $30.49964^{\circ}$, accuracy $7 \mathrm{~m}$;

1562 - 2017.05.02, Lisnyky, latitude $50.29778^{\circ}$, longitude $30.54402^{\circ}$, accuracy $6 \mathrm{~m}$;

1555 - 2017.05.02, Lisnyky, latitude $50.29575^{\circ}$, longitude $30.53792^{\circ}$, accuracy $4 \mathrm{~m}$;

1559 - 2017.05.02, Lisnyky, latitude $50.29609^{\circ}$, longitude $30.53963^{\circ}$, accuracy $4 \mathrm{~m}$;

1558 - 2017.05.02, Lisnyky, latitude $50.29611^{\circ}$, longitude $30.53952^{\circ}$, accuracy $4 \mathrm{~m}$;

1556 - 2017.05.02, Lisnyky, latitude $50.29610^{\circ}$, longitude $30.53956^{\circ}$, accuracy $5 \mathrm{~m}$;

1586 - 2018.05.05, Sviatoshynske forestry, latitude $50.49111^{\circ}$, longitude $30.31022^{\circ}$, accuracy $6 \mathrm{~m}$;

1532 - 2017.04.30, Holosiivskyi wood, latitude $50.37271^{\circ}$, longitude $30.49991^{\circ}$, accuracy $5 \mathrm{~m}$. 


\section{References}

Boiko M.F. 2008. Cheklist mokhopodibnykh Ukrayiny. Kherson: Aylant, 229 рр. [Бойко М.Ф. 2008. Чекліст мохоподібних Украӥни. Херсон: Айлант, 229 с.].

Gapon S.V. 2009. Ukrainian Botanical Journal, 66(4): 477-488. [Гапон С.В. 2009. Епіфітні бріоугруповання ландшафтного заказника "Чорноліський" (Кіровоградська обл.). Український ботанічний журнал, 66(4): 477-488].

Gapon S.V. 2012. Chornomors'kyi Botanichnyi Zhurnal, 8(2): 214-221. [Гапон С.В. 2012. Бріофлора і мохова рослинність національних природних парків Лісостепу України. Чорноморський ботанічний журнал, 8(2): 214-221].

Gapon S.V. 2014. Syntaksonomiia mokhovoi roslynnosti Ukrainy (Lisostep). Poltava: FOP Kulibaba, 88 pp. [Гапон С.В. 2014. Синтаксономія мохової рослинності України (Лісостеп). Полтава: ФОП Кулібаба, 88 с.].

Gapon S.V. 2017. Biolohia ta Ekolohiia, 3(1-2): 14-19. [Гапон С.В. 2017. Природно-заповідні об'єкти як осередки збереження бріорізноманіття в умовах України. Біологія та екологія, 3(1-2): 14-19].

Gapon S.V., Gapon Yu.V. 2018. Biolohiia ta Ekolohiia, 4(1): 17-26 [Гапон С.В., Гапон Ю.В. 2018. Сучасна класифікаційна схема мохової рослинності України. Біологія та екологія, 4(1): 17-26.].

Gapon Yu.V. 2015. Visnyk Problem Biolohii i Medytsyny, 4(2): 71-73. [Гапон Ю.В. 2015. Мохоподібні на мохова рослинність лісових масивів НПП "Нижньосульський"
(Полтавська обл.). Вісник проблем біології і медицини, 4(2): 71-73].

Gapon Yu.V. 2017. Visnyk Problem Biolohii i Medytsyny, 3(1): 76-81. [Гапон Ю.В. 2017. Мохова рослинність міст Роменсько-Полтавського геоботанічного округу. Вісник проблем біологї і медицини, 3(1): 76-81].

Hugonnot V., Celle J. 2013. The Leptodictyo riparii-Hygroamblystegietum varii ass. nov., a dead wood dwelling association of near-natural alluvial forests in the Rhône valley (France). Herzogia, 26(1): 187-195.

Marstaller R. 2006. Syntaxonomischer Konspekt der Moosgesellschaften Europas und angrenzender Gebite. Haussknechtia, 13: 1-192.

Mucina L., Bultmann H., Dierßen K., Theurillat J.-P., Raus T., Čarni A., Šumberová K., Willner W., Dengler J., Gavilán García R., Chytrý M., Hájek M., Di Pietro R., Iakushenko D., Pallas J., Daniëls F.J.A., Bergmeier E., Santos Guerra A., Ermakov N., Valachovič M., Schaminée J.H.J., Lysenko T., Didukh Y.P., Pignatti S., Rodwell J.S., Capelo J., Weber H.E., Solomeshch A., Dimopoulos P., Aguiar C., Hennekens S.M., Tichý L. 2016. Vegetation of Europe: hierarchical floristic classification system of vascular plant, bryophyte, lichen, and algal communities. Applied Vegetation Science, 19 (Suppl. 1): 3-264.

Virchenko V.M., Gapon S.V. 2009. Zhyva Ukraina, 1-2: 8. [Вірченко В.М., Гапон С.В. 2009. Мохоподібні урочища "Феофанія". Жива Україна, 1-2: 8].

Recommended for publication by S.Ya. Kondratyuk 